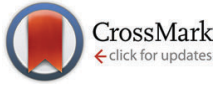

Cite this: Chem. Commun., 2016, 52,5132

Received 15th January 2016,

Accepted 10th March 2016

DOI: $10.1039 / c 6 c c 00403 b$

www.rsc.org/chemcomm

\section{Fastest non-ionic azo dyes and transfer of their thermal isomerisation kinetics into liquid-crystalline materials $\dagger$}

\author{
Jaume Garcia-Amorós, ${ }^{a}$ M. Cidália R. Castro, ${ }^{b}$ Paulo Coelho, ${ }^{c}$ \\ M. Manuela M. Raposo*b and Dolores Velasco*a
}

Push-pull bithienylpyrrole-based azo dyes exhibit thermal isomerisation rates as fast as $1.4 \mu \mathrm{s}$ in acetonitrile at $298 \mathrm{~K}$ becoming, thus, the fastest neutral azo dyes reported so far. These remarkably low relaxation times can be transferred into liquid-crystalline matrices enabling light-triggered oscillations in the optical density of the final material up to $11 \mathrm{kHz}$ under ambient conditions.

Liquid crystals (LCs) find numerous applications in a diversity of areas, including LC displays, ${ }^{1,2}$ enantioselective synthesis, ${ }^{3,4}$ medical thermography, ${ }^{5,6}$ analytical chemistry ${ }^{7,8}$ and artificial muscle-like actuation, ${ }^{9-12}$ mainly because of the ability of the mesogenic molecules to change their orientation in response to relatively weak physical and chemical cues. In particular, light is an especially attractive trigger to tune the macroscopic properties of liquid-crystalline materials on demand since it can be delivered with superior spatiotemporal resolution enabling, moreover, in situ monitoring of the whole operation in real time. However, for such purpose to be achieved either the mesogenic molecules should be photoactive or the material must be doped with convenient light-sensitive molecules.

Development of synthetic chemistry has yielded over the years a wide palette of organic photochromes that could be suitable for this task. In this context, the reversible photochemical interconversion between the trans and cis isomers of azobenzene, in combination with the possibility of its cis form to revert to the initial state thermally in the dark ${ }^{13}$ has rendered

\footnotetext{
${ }^{a}$ Grup de Materials Orgànics, Institut de Nanociència i Nanotecnologia $\left(I N^{2} U B\right)$, Departament de Química Orgànica, Universitat de Barcelona, Martí i Franqués 1, E-08028, Barcelona, Spain. E-mail: dvelasco@ub.edu; Fax: +34 9333978 78; Tel: +34934039260

${ }^{b}$ Centro de Quimica, Universidade do Minho, Campus de Gualtar, 4710-057, Braga, Portugal. E-mail: mfox@quimica.uminho.pt; Fax: +3516043 82;

azo dyes as the photochromes of common choice for lightcontrolled applications within materials science.

Controlled switching of molecular function requires the design and synthesis of appropriate molecular photochromic systems and a basic understanding of their structural and electronic properties including the interaction with the environment. ${ }^{14}$ Recently, we have reported a wide variety of very fast isomerising cationic azo dyes with relaxation times ranging from the millisecond to the nanosecond temporal domain. ${ }^{15}$ However, the vast majority of research into the photo-responsive properties of these push-pull systems has been limited to the solution phase, mainly because of the low solubility of such organic molecules in conventional host matrices due to their ionic nature. This feature limits substantially their final applicability. Moreover, although the thermal back isomerization of conventional and push-pull type azo dyes in nematic LCs has been intensively investigated during the last decade, ${ }^{16-18}$ azo dye-based photoactive LC mixtures isomerizing within the microsecond timescale have not been achieved. On this basis, the design of novel fast isomerising azo chromophores and their further integration into either flexible polymeric architectures or solid solutions that will not negatively impact the response time of the photoresponsive component are in great demand.

We report herein on the synthesis and thermal isomerisation of a new family of bithienylpyrrole-based push-pull azo dyes (Fig. 1) that show thermal isomerisation rates as fast as $1 \mu \mathrm{s}$ at $298 \mathrm{~K}$. In addition, we have explored their integration into two types of well-known host matrices, isotropic PMMAbased polymers and nematic LCs, in order to obtain thin film materials exhibiting quick oscillations in their optical density under ambient conditions.

Azo dye 2a has been prepared as reported elsewhere. ${ }^{19}$ Compounds 1a, 3a-5a and 3b-4b (Fig. 1) were synthesized in moderate to good yields (22-66\%) via the diazotization of 4-cyanoaniline, 2-cyano-4-nitroaniline, 4-cyano-2-nitroaniline and 2,4-dinitroaniline with $\mathrm{NaNO}_{2}$ in either $\mathrm{HCl}$ or $\mathrm{H}_{2} \mathrm{SO}_{4}$ (5a) at $0-5{ }^{\circ} \mathrm{C}$ and further coupling with bithienylpyrrole or 


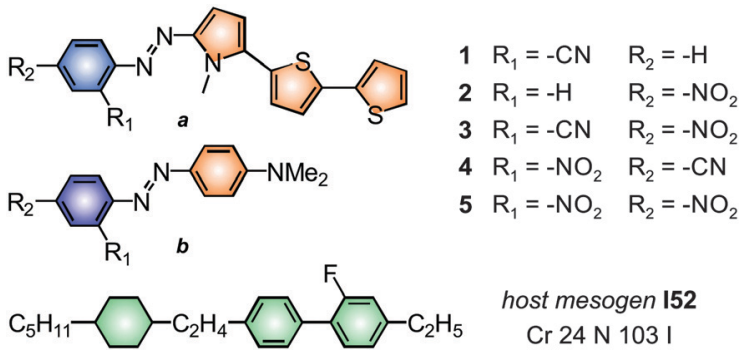

Fig. 1 Chemical structure of azo dyes $1 a-5 a$ and $3 b-4 b$, and of the nematic mesogen I52. Phase transition temperatures are given in Celsius.

4 -amino- $N, N$-dimethylaniline in acetonitrile or in a $1: 1(\mathrm{v} / \mathrm{v})$ mixture of acetic anhydride and ice water $(\mathbf{5 a})$ at $0{ }^{\circ} \mathrm{C}$ (Fig. S1 and S2, ESI $\dagger$ ). ESIMS together with ${ }^{1} \mathrm{H}$ and ${ }^{13} \mathrm{C}$ NMR spectra confirmed their structural identities.

The kinetics of the thermal cis-to-trans isomerisation for azo dyes $\mathbf{1 a}-\mathbf{5 a}, \mathbf{3 b}$ and $\mathbf{4 b}$ dissolved in both isotropic (acetonitrile, ethanol, toluene and 1,2-dimethoxyethane) and nematic (I52 in Fig. 1) solvents was analysed by means of the nanosecond laser flash-photolysis technique (ESI $\dagger$ ). The relaxation time of the cis isomers ( $\tau$ in Table $1, \tau=1 / k)$ was determined by fitting a single exponential function to the data. The maximum oscillation frequency $\left(\nu_{\mathrm{Max}}\right.$ in Table 1) in the optical density of the final material was determined as $\nu_{\operatorname{Max}}=1 /(3 \tau)$.

Parent azo derivatives 1a and 2a, which contain cyano and nitro functions in the ortho and para position, relative to the azo function, respectively, display relaxation times for their thermal back reaction within the millisecond temporal domain (49 $\mathrm{ms}$ and $1.6 \mathrm{~ms}$ in ethanol at $298 \mathrm{~K}$, respectively). The combination of these two well-known electron-withdrawing groups in the same aromatic ring (compounds 3a and $\mathbf{4 a}$ in Fig. 1) translates in a dramatic acceleration of the thermal isomerisation of the azo photochrome registering thereby relaxation times within the microsecond timescale (3.1 and $74 \mu$ s for $3 \mathbf{a}$ and $4 a$, respectively, a and c in Fig. 2). The replacement of the cyano function of $\mathbf{3 a}$ and $4 \mathbf{a}$ by a second nitro substituent (5a in Fig. 1) yielded an isomerisation rate as fast as that of its 2-cyano-4-nitro-substituted counterpart 3a (4.1 $\mu \mathrm{s} v s .3 .1 \mu \mathrm{s}$ in ethanol). Thus, the presence of a nitro group in the para position, relative to the azo function, results in faster thermal isomerization kinetics. In addition, azo dyes $\mathbf{3 a}$ and 5a exhibit relaxation times of only 1.4 and $1.7 \mu \mathrm{s}$ in

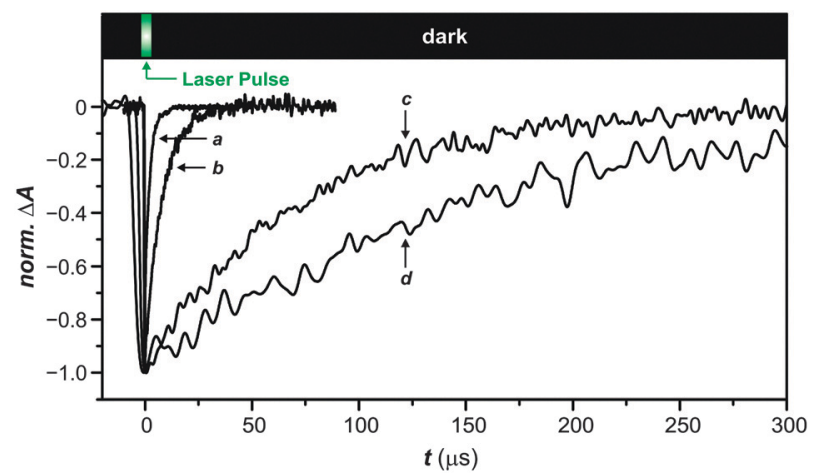

Fig. 2 Transient absorption change photoinduced by laser pulsed irradiation with green light (Nd-YAG laser, $5 \mathrm{~ns}$ pulse width, $10 \mathrm{~mJ}$ per pulse, $\lambda_{\text {irrad }}=532 \mathrm{~nm}, \lambda_{\text {obs }}=500 \mathrm{~nm}$ ) for azo compounds $3 \mathbf{a}(\mathrm{a}), \mathbf{3 b}(\mathrm{b}), \mathbf{4 a}(\mathrm{c})$ and $\mathbf{4 b}(\mathrm{d})$ in ethanol at $298 \mathrm{~K}([\mathrm{AZO}]=10-20 \mu \mathrm{M})$

acetonitrile at $298 \mathrm{~K}$, being, thus, the fastest thermally isomerising neutral azo derivatives described heretofore.

The ability of these azo derivatives to dissolve readily in PMMA thereby forming light-sensitive homogeneous thin films might make such organic chromophores valuable candidates to achieve fast oscillation frequencies in the solid state, where no solvent is present. However, the isomerisation rate for the azo dyes studied decreased dramatically when immersed in PMMA polymeric matrices. Indeed, the relaxation time of azo compounds 3-5 increased from the microsecond temporal domain up to several seconds (3a and 4a, Fig. S5 and S6, respectively, ESI $\dagger$ ) or minutes (1a, Fig. S4, ESI $\dagger$ ). Therefore, the rigidness imposed by the PMMA backbone precludes the transfer of the low response time displayed by these photoactive molecular constructs in isotropic solvents to the solid state.

A promising strategy to overcome this drawback can be the use of liquid-crystalline host systems. Indeed, the rod-like shape of the trans isomer of azo dyes 1-5 enables their integration as doped guests into this particular class of selforganized materials. Remarkably, the thermal isomerisation rate for azo derivatives $3 \mathbf{a}$ and $\mathbf{5 a}$ in the viscous nematic phase of mesogen $\mathbf{I 5 2}$ ( 30 and $70 \mu \mathrm{s}$, respectively) falls within the same temporal domain than those in highly polar conventional isotropic solvents, like acetonitrile $(\varepsilon=37.5)$ and ethanol $(\varepsilon=24.5)$, but they are $c a .200$-fold faster than those in toluene, which exhibits a polarity similar to that of mesogen $\mathbf{I 5 2}$ (2.4 vs. 2.9, respectively).

Table 1 Kinetic $(\tau)$ and thermal activation parameters $\left(\Delta H^{\neq}\right.$and $\left.\Delta S^{\neq}\right)$for the cis-to-trans isomerisation, and maximum oscillation frequency $\left(\nu_{\text {max }}\right)$ for azo dyes $\mathbf{1} \mathbf{a}-\mathbf{5 a}$ and $\mathbf{3 b}-\mathbf{4 b}$ in different media at $298 \mathrm{~K} . \tau, \Delta H^{\neq}, \Delta \mathrm{S}^{\neq}$and $\nu_{\max }$ are given in $\mu \mathrm{s}, \mathrm{kJ} \mathrm{mol}^{-1}, \mathrm{~J} \mathrm{~K}^{-1} \mathrm{~mol}^{-1}$ and kHz, respectively

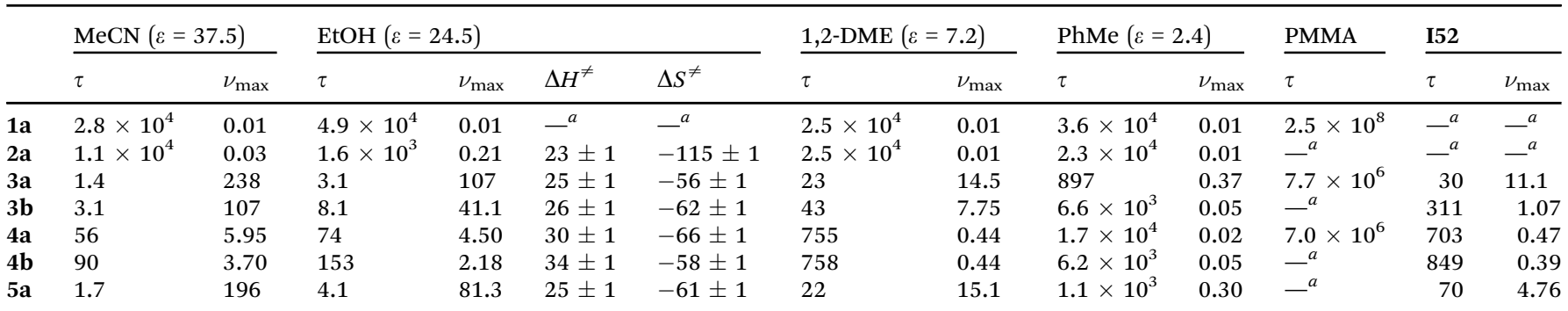

${ }^{a}$ Not determined. 

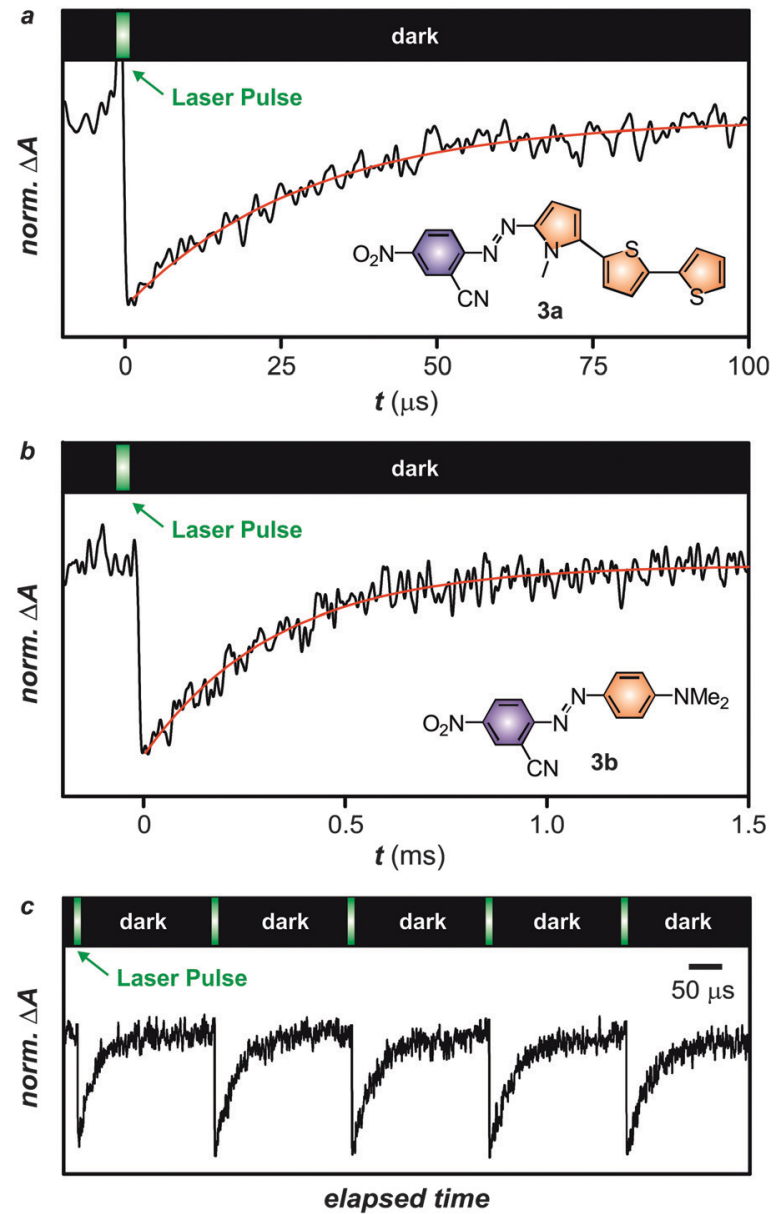

Fig. 3 Transient absorption change photoinduced by laser pulsed irradiation with green light (Nd-YAG laser, $5 \mathrm{~ns}$ pulse width, $10 \mathrm{~mJ}$ per pulse, $\lambda_{\text {irrad }}=532 \mathrm{~nm}, \lambda_{\text {obs }}=500 \mathrm{~nm},[A Z O]=4 \mathrm{mM}$ ) for azo compounds $3 \mathrm{a}$ (a) and $\mathbf{3 b}$ (b) in the nematic mesophase of $\mathbf{1 5 2}$ at $298 \mathrm{~K}$, and oscillation in the optical density (c) of azo dye 3a under the very same experimental conditions.

On this basis, such drastic enhancement of the isomerisation rate should be ascribed to the influence of the nematic mean field on the thermal recovery of the thermodynamically stable trans form of the azo photochome.

It is well known that illumination of azo dye-doped nematic mixtures results in a dramatic misalignment of the mesogens. ${ }^{20}$ Once the irradiation ceases, the tendency of the LC molecules to recover their initial orientation enhances the isomerisation rate of the azo dye through a cooperative interaction. ${ }^{21}$ Accordingly, such a synergistic effect translates into a less enthalpic term and a much more negative $\Delta S^{\neq}$contribution in the nematic mesogen $\mathbf{I 5 2}$ than in toluene (Table S1, ESI $\dagger$ ). Moreover, this interaction becomes stronger for those azo dyes displaying bithienylpyrrole electron-donating platforms within their covalent skeleton, due to their more extended rod-like geometry, showing thereby much faster relaxation times than their amino-benzenebased counterparts. Specifically, bithienylpyrrole-containing azo dye 3a, which exhibits a thermal relaxation time of only $30 \mu \mathrm{s}$ at $298 \mathrm{~K}$ when doped in the host nematic LC I52 (Fig. 3a), displays a 10-fold faster isomerisation kinetics than its corresponding amino-benzenebased analogue $\mathbf{3 b}$ (Fig. 3b). In this way, the introduction of azo dyes 1-5 as doped guests in self-organized nematic phases compensates the drastic deaccelerating effect imposed by the intrinsic rigidness of the PMMA polymeric matrix due to the strong tendency of their constituting mesogenic molecules to be realigned along the director direction once the illumination of the samples stops.

Finally, the photostability of the different LC-based photochromic molecular switches was tested by subjecting them to several pulsed green light (532 nm)-dark cycles. Fig. 3c evidences the high photo-stability exhibited by the bithienylpyrrole-based azo dye 3a in the nematic host $\mathbf{I 5 2}$ at $298 \mathrm{~K}$. Specifically, this particular mixture permits optical oscillations in the optical density of the system up to $11 \mathrm{kHz}$ under ambient conditions. Moreover, after 500 cycles neither the photoinduced absorbance variation nor the relaxation time of the molecular switch was altered by its continuous work. A similar behaviour was registered for the different azo dyes analysed.

Push-pull bithienylpyrrole-based azo compounds are the fastest neutral thermally isomerising azo dyes reported so far. Specifically, these photoactive systems display thermal isomerisation kinetics as fast as $1 \mu \mathrm{s}$ at $298 \mathrm{~K}$ when dissolved in conventional organic solvents. Remarkably, the highly extended rod-like shape of these particular chromophores makes them excellent guests to be introduced in host nematic mesophases. Indeed, the effective non-covalent interaction between the azo dyes and the nematogen molecules enables the transfer of the thermal isomerisation rate of the former into the self-organized host mesophase, due to the great tendency of the latter to recover their initial orientation along the director after irradiation. Thus, nematic LC mixtures containing bithienylpyrrole-based azo dyes as doped guests permit optical oscillations in the optical density of the system up to $11 \mathrm{kHz}$ under ambient conditions being, therefore, the quickest LC optical oscillators described so far.

Financial support for this research was obtained from the Ministerio de Economía y Competitividad (Spain, CTQ2012-36074 and CTQ2015-65770-P). The authors thank Prof. Santi Nonell for his help with the flash photolysis measurements. Thanks are also due to: Fundação para a Ciência e Tecnologia (FCT) for a PhD grant to M. C. R. Castro (SFRH/BD/78037/2011); FEDERCOMPETE for financial support through the Centro de Química UM, PEst-C/QUI/UI0686/2013 (FCOMP-01-0124-FEDER-037302). The NMR spectrometer Bruker Avance III 400 is part of the National NMR Network and was purchased within the framework of the National Program for Scientific Re-equipment, contract REDE/1517/RMN/2005 with funds from POCI 2010 (FEDER) and FCT.

\section{References}

1 G. W. Gray, Handbook of Liquid Crystals, Wiley-VCH, Weinheim, 1998.

2 D.-K. Yang and S.-T. Wu, Fundamentals of Liquid Crystal Devices, Wiley-VCH, Chichester, 2015.

3 Y. Ishida, Y. Kai, S.-Y. Kato, A. Misawa, S. Amano, Y. Matsuoka and K. Saigo, Angew. Chem., Int. Ed., 2008, 120, 8365-8369.

4 Y. Xu, G. Yang, H. Xia, G. Zou, Q. Zhang and J. Gao, Nat. Commun., $2014,5,5050$. 
5 J. Stasiek, M. Jewartowski and T. A. Kowalewski, J. Cryst. Process Technol., 2014, 4, 46-59.

6 S. J. Woltman, G. D. Jay and G. P. Crafword, Liquid Crystals: Frontiers in Biomedical Applications, World Scientific Publishing Co., London, 2007.

7 F. Gritti and G. Félix, Chromatographia, 2002, 55, 523-531.

8 H. J. Issaq, A Century of Separation Science, Marcel Dekker Inc., New York, 2002.

9 (a) H. Wermter and H. Finkelmann, e-Polym., 2001, 13, 1-13; (b) M. Camacho-López, H. Finkelmann, P. Palffy-Muhoray and M. Shelley, Nat. Mater., 2004, 3, 307-310.

10 (a) C. M. Spillmann, J. Naciri, M.-S. Chen, A. Srinivasan and B. R. Ratna, Liq. Cryst., 2006, 33, 373-380; (b) C. M. Spillmann, J. Naciri, B. D. Martin, W. Farahat, H. Herr and B. R. Ratna, Sens. Actuators, A, 2007, 133, 500-505.

11 (a) J. Garcia-Amorós, H. Finkelmann and D. Velasco, J. Mater. Chem., 2011, 21, 1094-1101; (b) J. Garcia-Amorós, M. Martínez, H. Finkelmann and D. Velasco, Phys. Chem. Chem. Phys., 2014, 16, 8448-8454.

12 (a) M. Yamada, M. Kondo, J.-I. Mamiya, Y. Yu, M. Kinoshita, C. J. Barrett and T. Ikeda, Angew. Chem., Int. Ed., 2008, 47, 4986-4988; (b) H. Yu and T. Ikeda, Adv. Mater., 2011, 23, 2149-2180; (c) A. Priimagi, A. Shimamura, M. Kondo, T. Hiraoka, S. Kubo, J.-I. Mamiya, M. Kinoshita, T. Ikeda and A. Shishido, ACS Macro Lett., 2012, 1, 96-99.
13 H. Rau, Photochemistry and Photophysics, CRC Press, Boca Raton, FL, 1990.

14 (a) J. Garcia-Amorós and D. Velasco, Phys. Chem. Chem. Phys., 2014, 16, 3108-3114; (b) J. Garcia-Amorós, M. Reig, M. C. R. Castro, A. Cuadrado, M. M. M. Raposo and D. Velasco, Chem. Commun., 2014, 50, 6704-6706.

15 (a) J. Garcia-Amorós, W. A. Massad, S. Nonell and D. Velasco, Org. Lett., 2010, 12, 3514-3517; (b) J. Garcia-Amorós, S. Nonell and D. Velasco, Chem. Commun., 2011, 47, 4022-4024; (c) J. GarciaAmorós, S. Nonell and D. Velasco, Chem. Commun., 2012, 48, 3421-3423; (d) J. Garcia-Amorós, A. Bucinskas, M. Reig, S. Nonell and D. Velasco, J. Mater. Chem. C, 2014, 2, 474-480.

16 T. Ikeda and O. Tsutsumi, Science, 1995, 268, 1873-1875.

17 O. Tsutsumi, Y. Demachi, A. Kanazawa, T. Shiono, T. Ikeda and Y. Nagase, J. Phys. Chem. B, 1998, 102, 2869-2874.

18 O. Tsutsumi, A. Kanazawa, T. Shiono, T. Ikeda and L.-S. Park, Phys. Chem. Chem. Phys., 1999, 1, 4219-4224.

19 J. Garcia-Amorós, M. C. R. Castro, P. Coelho, M. M. M. Raposo and D. Velasco, Chem. Commun., 2013, 49, 11427-11429.

20 J. H. Sung, S. Hirano, O. Tsutsumi, A. Kazanawa, T. Shiono and T. Ikeda, Chem. Mater., 2002, 14, 385-391.

21 (a) J. Garcia-Amorós, M. Martínez, H. Finkelmann and D. Velasco, J. Phys. Chem. B, 2010, 114, 1287-1293; (b) J. Garcia-Amorós, H. Finkelmann and D. Velasco, Phys. Chem. Chem. Phys., 2011, 13, 11233-11238. 